\title{
Correction: Macroevolution of gastric Helicobacter species unveils interspecies admixture and time of divergence
}

\author{
Annemieke Smet ${ }^{1,2} \cdot$ Koji Yahara $^{3} \cdot$ Mirko Rossi $^{4} \cdot$ Alfred Tay $^{5} \cdot$ Steffen Backert ${ }^{6} \cdot$ Armin Ensser $^{7} \cdot$ James G. Fox $^{8}$. \\ Bram Flahou $^{2} \cdot$ Richard Ducatelle $^{2} \cdot$ Freddy Haesebrouck $^{2} \cdot$ Jukka Corander $^{9,10,11}$
}

Published online: 28 February 2019

(c) International Society for Microbial Ecology 2019

\section{Correction to: ISME J. 2018;12:2518-2531 https://doi.org/10.1038/s41396-018-0199-5; published online 25 June 2018}

The original version of this Article contained an error in the presentation of the author Armin Ensser, which was incorrectly given as Ensser Armin. The correct author list is as follows:

Annemieke Smet, Koji Yahara, Mirko Rossi, Alfred Tay, Steffen Backert, Armin Ensser, James G. Fox, Bram Flahou, Richard Ducatelle, Freddy Haesebrouck, Jukka Corander

The authors apologise for any inconvenience caused.
These authors contributed equally: Annemieke Smet, Koji Yahara, Mirko Rossi

These authors jointly supervised this work: Freddy Haesebrouck, Jukka Corander

The original article can be found online at https://doi.org/10.1038/ s41396-018-0199-5.

Annemieke Smet

annemieke.smet@uantwerpen.be

$\triangle$ Koji Yahara

k-yahara@nih.go.jp

$\triangle$ Mirko Rossi

mirko.rossi@helsinki.fi

1 Laboratory Experimental Medicine and Pediatrics, Faculty of Medicine and Health Sciences, University of Antwerp,

Antwerp, Belgium

2 Department of Pathology, Bacteriology and Avian Diseases, Faculty of Veterinary Medicine, Ghent University, Merelbeke, Belgium

3 Antimicrobial Resistance Research Center, National Institute of Infectious Diseases, Tokyo, Japan

4 Department of Food Hygiene and Environmental Health, Faculty of Veterinary Medicine, University of Helsinki, Helsinki, Finland
The Marshall Centre for Infectious Diseases Research and Training, School of Pathology and Laboratory Medicine, University of Western Australia, Nedlands, Perth, WA, Australia

6 Division Microbiology, Department Biology, University Erlangen Nuremberg, Erlangen, Germany

7 Institute of clinical and Molecular Virology, Universitätsklinikum Erlangen, Erlangen, Germany

8 Division of Comparative Medicine, Massachusetts Institute of Technology, Cambridge, MA, USA

9 Department of Biostatistics, University of Oslo, Oslo, Norway

10 Department of Mathematics and Statistics, University of Helsinki, Helsinki, Finland

11 Welcome Trust Sanger Institute, Cambridge, UK 